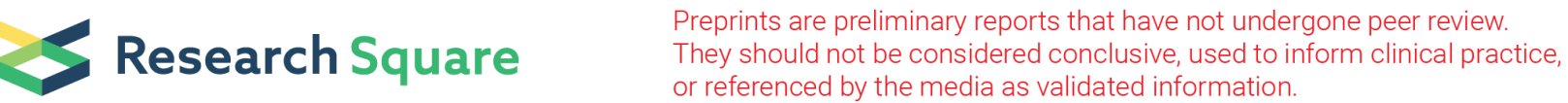

\section{Preferences and Expectations of Feedback of Individual Genetic Research Results in African Genomics: Views of South African Parents of Children With Neurodevelopmental Conditions}

Olivia Precious Matshabane ( $\square$ olivia.matshabane@uct.ac.za )

University of Cape Town https://orcid.org/0000-0002-5977-8904

Cleo A Albertus

University of Cape Town Faculty of Health Sciences

Marlyn C Faure

University of Cape Town Faculty of Health Sciences

Dimpho Ralefala

University of Cape Town Faculty of Health Sciences

Kirsten A Donald

University of Cape Town Institute of Child Health: University of Cape Town Department of Paediatrics and Child Health

Ambroise Wonkam

University of Cape Town Faculty of Health Sciences

Jantina de Vries

University of Cape Town Faculty of Health Sciences

Research article

Keywords: African Genomics, Individual Findings, Feedback of Findings, Parents of children, Neurogenetics research

Posted Date: November 23rd, 2020

DOl: https://doi.org/10.21203/rs.3.rs-107280/v1

License: (c) (1) This work is licensed under a Creative Commons Attribution 4.0 International License.

Read Full License 


\section{Abstract}

\section{Background}

Genomic medicine is expanding at an exponential pace across the globe and increased access to genome analysis has led to greater generations of genetic results with specific relevance to individuals.

Aim

This study aims to explore preferences and expectations of feedback of individual genetic research results among parents of children with neurodevelopmental conditions.

Methods

Following a qualitative approach, we conducted four deliberative focus group discussions with $(n=27)$ South African parents of children involved in genomics research on neurodevelopmental conditions.

Results

Most participants expressed a strong interest in receiving individual genetic results regardless of severity, actionability and preventability. These results were viewed as valuable because they could empower or emancipate individuals, families and communities. Receiving risk information was also believed to motivate healthier lifestyle choices. However, some participants were uncertain or articulated a desire not to receive results due to fears of anxiety or psychological harm. In addition, participants expected to receive results as a demonstration of respect from researchers and articulated it as an act to build trust between researchers and participants.

\section{Conclusions}

Internationally, a debate continues around whether individual genetic results should or should not be fed back to participants of research studies. In Africa, there is scant literature which has investigated this question and no policies to guide researchers. This study provides a basis of empirical data on perspectives of African participants which could inform work on the development of a consolidated approach to the feedback of incidental findings in the continent.

\section{Background}

Genomic medicine is expanding at a rapid pace across the globe. The aim of genomic research is to significantly improve therapeutic approaches and interventions in order to efficiently understand disease mechanisms, while moving towards personalised medicine. Genomic studies have the potential to identify genomic research results that are relevant to individuals. With the increase of genomic research on the African continent, an important ethical question is whether genomics researchers ought to return selected individual research results to study participants. Given that the main purpose of genomics 
research is usually to obtain generalisable information, which is relevant to populations and not individuals, returning individual results can be perceived to be going beyond that mandate. Despite such concerns, scholars from high income countries have agreed on specific results which must, should, may or must not be fed back (1). Results that ought to be fed back are for instance reflected in the American College of Medical Genetics and Genomics reported list which currently contains 59 actionable genes that are associated with 24 diseases, for which preventative or curative measures are possible (2). Given the strong bias in the racial and the geographical locations of genomic data which such recommendations are based on (i.e. European populations from North America and Europe) (3), African scholars have suggested that there would likely need to be additional variants included in an Africaspecific list (4). Other scholars have suggested that despite this list not yet existing, it is important for African researchers to address the question of feeding back genomic results which are currently being uncovered and developing consensus guidelines to advise the process of return of genomic results in Africa (5). As such, on the basis of collaborative efforts, the Human Heredity and Health in Africa (H3Africa) consortia has developed an evolving document on recommendations for the feedback of individual genetic research results for H3Africa projects (6).

Globally, although views on returning genetic results vary among scientists (7) and the public (8), there is a generic interest in receiving feedback on genetic results (9). For example, a study led by scholars in the UK which investigated the attitudes of nearly 7000 health professionals, genomics researchers and members of the public from 75 different countries on the return of incidental results, found that the great majority of stakeholders felt that genomic research participants should receive their individual results. More specifically, $98 \%$ of their participants were personally interested in receiving results for conditions that may be preventable and/or life-threatening (9). This finding coincides with genomics research participants $(10,11)$ who have voiced an interest in being informed about their individual genetic risk, especially results that reveal an increased risk of preventable or treatable diseases, adverse medication reactions and carrier status for conditions with reproductive implications. In research conducted in Europe, $95 \%$ of genomics research participants indicated a strong preference to leaning all their individual genetic risk results, particularly results which are medically actionable or which signal carrier status (12). In Canada, Fermandez et al. (2013) found that $86 \%$ of parents of children enrolled in cancer genomics research emphasised a right to receiving incidental results. A majority of parents in their study also wanted results predicting an increased risk of untreatable fatal conditions $(83 \%)$, multiple conditions $(87 \%)$ and conditions with uncertain impact (70\%) (13).

Limited literature documents the views of marginalised groups in research, however there is some evidence suggesting that marginalised groups from the global north have differing views. Most recently, in the US, Sabatello, Zhang, Chen and Appelbaum (2020) found that interest in receiving biological and health-related individual genetic results from precision medicine research varied significantly across gender, race/ethnicity and disability groups (14). In their study, most participants reported an interest in receiving genetic risk results about 'a treatable disease', like asthma (68\%) and 'an untreatable disease' like Alzheimer's (61\%) (14). Also in the US, Lakes and colleagues emphasised that perceptions and attitudes about feedback of findings may be different for individuals of different racial and cultural 
backgrounds (15) and that individuals view return of results as a complex issue which is compounded by individual and cultural differences. For instance, in their study with Hispanic, White and Asian American participants they found that participants desired a dynamic, flexible process that accommodated individual and contextual factors in relation to returning of genetic results (15). Currently, there is sparse empirical evidence from the African continent investigating the views of genomic research participants and scientists on the return of individual genetic results $(4,16)$, whilst there is arguably a strong imperative to return research results (17).

Against this background, this study aims to explore the preferences for and expectations regarding the feedback of individual genetic findings among South African parents of children with neurodevelopmental conditions involved in genomics research.

\section{Methods}

\section{Sampling and data collection}

Four deliberative focus group discussions (dFGDs) involving parents of children involved in genomics research on neurodevelopmental conditions, were held in a public children's hospital in Cape Town, South Africa. The participants had enrolled in a larger genetics study focusing on neurodevelopmental conditions (NeuroDEV) (18) where children and parents provided blood samples for exome sequencing. Each dFGD group consisted of 4 - 8 participants varying in age, gender, level of education, experience of engaging with genetic research, religion and culture.

We enrolled 27 participants, 14 from the Xhosa population and 13 from the Mixed-ancestry population in South Africa. Among the Xhosa parents, we enrolled 12 (82\%) mothers and $2(18 \%)$ fathers, 7 (50\%) of them being married and with a mean age of 39.23 years. Eleven $(78.57 \%)$ had secondary schooling and $12(85.72 \%)$ were unemployed or employed in low-paid jobs. Among the Mixed-ancestry parents, we enrolled 7 (53.85\%) (fathers) and 6 (46.15\%) mothers, 11 (84.62\%) were married and they had a mean age of 37.67 years. Nine $(69.23 \%)$ had secondary schooling and $9(69 \%)$ were unemployed or employed in low-paid jobs. Overall, we had an $88.89 \%$ return rate for the second dFGDs. We endeavoured to establish diversity among participants as an attempt to ensure a range of different perspectives were collected.

The data collection procedure included three separate activities in the groups and was guided by a manual developed for this study. The first phase involved an information session (which took roughly 1.5 hours) to: 1.) provide an introduction and rationale for why the research study is being undertaken followed by a consenting process, and 2.) through the use of a brochure and an animated video we presented participants with some basic information about genetics and genomics research and the link between genetics and health. Following a short break, a second session (which lasted about 1.5 hours) was held with each group to: 3.) present case study scenarios and embedded questions prompting participants to deliberate on when and how individual results should be fedback and 4.) provide a debrief and closing. The following week participants returned for phase 2 which involved another 1-hour session 
where we began by recalling and further deliberating on some of the responses and questions provided by participants during the first dFGD. Then we delved deeper into the types of conditions for which the participants would like to receive results. The types of conditions discussed considered severity and preventability in accordance with a table developed by Holm and colleagues (19). The table includes examples of conditions that are not severe and preventable like an iron deficiency, severe and preventable like asthma, not severe and non-preventable like poor vision and severe and non-preventable like Alzheimer's disease. During this phase 2 session the facilitator and co-facilitator continued to engage participants in a deliberation of which categories of results they would like to receive from research as well as why they would like those results. Overall, each dFGD group engaged in deliberations guided by the facilitator and co-facilitator for approximately 4-hours over the duration of the two sessions.

\section{Data management and analysis}

DFGDs were recorded and the audiotapes were transcribed with the texts stored in a password protected computer. The participants' names were not used, instead they were given numbers to identify themselves. Recordings of dFGDs conducted in isiXhosa were transcribed and translated into English by a professional transcriber and translator. DFGD data were analysed using a Framework Approach to data analysis, which uses matrices to organize data, and which allows for the concurrent analysis of themes in the data (20). The framework analysis approach combines conventional inductive thematic analysis of qualitative data with a systematic approach to visualizing and analyzing the data $(20,21)$. We used the NVIV012 software package which fully supports the framework analysis (22). The analytical process involved transcription, data familiarization, so-called 'open coding' followed by more hierarchical or thematic codes, developing a working analytical framework, applying the analytical framework, charting data into framework matrix and interpreting the data (20).

\section{Ethics review}

Ethics approval was obtained from UCT's Faculty of Health Sciences Human Research Ethics Committee prior to commencement of the study (FHS: 782/2018). Research took place in accordance with UCT's Research Ethics Policies, the Declaration of Helsinki (Version 2013) and Good Clinical Practice guidelines. All participants in this study provided written informed consent.

\section{Results}

\section{Preferences for feedback of findings}

In this study we found that most participants wanted to receive individual genetic results across all four of Holms and colleagues's categories, regardless of severity, actionability and preventability. Only a few participants expressed uncertainty or a desire not to receive results and indicated their concern that receiving results may cause anxiety, particularly if the results were not medically actionable. Reasons for participants preferences are presented below. 


\section{Empowerment and Emancipation}

The overarching insight emerging during the dFGDs is that participants wanted to receive individual genetic results for a range of reasons, including "peace of mind", because receiving results would allow for an "ability to share findings with family and close people", to "reduce self-blame", to "preparing oneself financially and emotionally" and to "resolve uncertainty or self-blame". All participants spoke about the value of receiving health information from genomics research. For example,

"I feel information is power. The more information you have and even if you don't have the condition but the information you have might be able to help someone else..." (DFGD 4: Mixed ancestry parent)

Genetic risk information was generally seen as valuable for the parents themselves, their current children, potential future children and future generations. There was great value placed on sharing genetic risk information with family members in order for them to 'be alert' of any symptoms that may arise which are related to the condition they may be at increased risk of developing. See,

"Another thing, those results are very important. If there is a gene that they picked up, maybe in me or the child, which means that someone in the family can have that autism. Or if it could skip a generation, just keep the family alert, something like this could happen again. So, that is important." (P.1 DFGD 3: Xhosa parent)

In addition to the family being aware of the possibility for disease onset, some Xhosa participants expressed that knowing that result would also alleviate any misconceptions of the person being accused in their community of practicing witchcraft due to behaviours resulting from possible later symptoms of a mental condition like Alzheimer's. More specifically, having that information and sharing it with their family members ahead of time would allow for their family to be in a position to defend them or enlighten those who may be misinformed about the condition. For example,

Interviewer: "What if it is something serious like Alzheimer's, a condition that you will have in your late years. Now you are going to live all your life anticipating this disease..."

Parent: "I still would like to know so that people may know that there is a chance of me going crazy at age 60. Considering our culture and superstitions as Africans, people might say that I am a witch. So, it's better to be open about this. People are ignorant, they will say that I practice witchcraft. In a case where one is transparent, at least your family will know your condition." (DFGD 4: Xhosa parent)

In Xhosa culture, older women above the age of 50 who engage in unusual unexplainable behaviours are often accused of practicing witchcraft. These accusations can have a range of negative consequences for the victim and her family, inducing for instance social isolation, being ostracized or having their houses burnt down by community members (23). It is these anticipated negative experiences which 
prompted the participant to want their results for increased risk of a mental condition to be known by those around them.

Overall, most participants in this study expressed that they would like to receive genetic risk information because it would empower them with information and emancipate them through having 'peace of mind'. Furthermore, some participants shared that the information may reduce their self-blame for the onset of the condition. Some highlighted that receiving that information may help them to prepare emotionally and instrumentally by prompting them to invest in insurance policies and savings which could help them financially if/when the disease manifests.

\section{Motivation for healthy lifestyle}

Lifestyle choices, regular screening and alternative preventative therapies were the sub-themes which emerged under the overarching theme for motivation for a healthy lifestyle. Many participants framed their reason for wanting individual genetic results returned to be because the results may encourage them to make healthier "lifestyle choices", go for "regular screening", and seek "alternative preventative therapies" such as herbal medicine. For instance, one participant said,

"Yes, definitely tell her. You don't know the lifestyle that the lady is currently living, so if I would give her the results and then maybe she will change her lifestyle". (DFGD 4: Xhosa parent)

Another participant described how one could lead a healthier lifestyle after receiving genetic risk information. She said,

"Like if you know that in your family, there's cancer. So, eat differently. There are a lot of foods that causes cancer. Stop eating this food. You can always prevent it, so, ja. Definitely would want to know." (DFGD 2: Mixed Ancestry parent)

Another participant supported that view and alluded that having their genetic risk information may put them in a position where they can prevent the disease from manifesting or at least improve the prognosis of the disease. She said,

"I agree, I would also want to know. I think the best thing that you can do to, maybe, if you can prevent it, or better your future ... Like, if you get it, there are things that you can do to maybe make it not as bad, like he said, you can go for counselling, change your lifestyle etc. So, ja, I would want to know." (DFDG 2: Mixed Ancestry parent)

A few participants suggested that early knowledge of genetic risk information on conditions that may be considered untreatable through Western medicine may allow them to seek alternative healing or medicines which may help prevent or reduce the symptoms of the disease.

"The same with me. I would rather want to know, that maybe I can try and find alternative healing, or alternative way of medicine." (DFDG 2: Mixed Ancestry parent) 
In addition, some participants also expressed the view that receiving genetic risk information may allow for early Western medicine intervention, such as having a prophylactic mastectomy in the case of an increased risk of developing breast cancer. Overall, most participants talked about wanting to receive individual genetic results in order to encourage and promote a healthier lifestyle which could be preventative or lead to a delayed or less severe disease onset.

\section{REASONS FOR NOT WANTING FEEDBACK}

Whilst most participants indicated a strong preference for receiving individual genetic research results, a small number of participants were uncertain or expressed a desire not to know their results. Three participants indicated concerns and anxieties about the return of results. Participants expressed worry that receiving individual genetic results may cause psychological harm for the individual and possibly their family. For example, one participant said,

"Not knowing what to do to remedy the situation, a person could go on stressing unnecessarily." (DFGD 4: Xhosa parent)

Another participant recalled how receiving a label of depression from her mental health practitioner increased her anxiety. Reflecting on that experience, this participant highlighted that for her, getting the name of a condition, even if it is just an increased risk of getting it and not a diagnosis, would similarly cause overthinking and increase her anxiety. See,

"I will find out eventually, when I get there. For now, I just want to live my life. Like, if he didn't give the label, I would have still carried on like I did, before I knew... So, my mind works like that. It will just play too much on my mind." (DFDG 2: Mixed Ancestry parent)

Others were concerned whether results could lead to self-alienation and pessimism. See,

"If not knowing, I would just go on with my life. Because at the end of the day, when you have that actual news, your whole life changes completely... then maybe sometimes people will shut their whole life from the whole world. So, that's why I'm saying, yes and no. That's what I think, I'm just going to go with that. Not knowing..."(DFDG 2: Mixed Ancestry parent)

Another two participants changed their views during deliberations to not wanting to know their individual genetic results, they cited not being able to control or prevent everything in life and wanted to "let life take its course", so it seemed to them, better not to know.

"With or without this research, we are all sitting here. We all have the possibility of getting it. But right now, we could maybe have it in us already. Like, there's a possibility in us already. So, then why waste our time on all that? Just live. I think now I don't want to know any more." (DFDG 2: Mixed Ancestry parent)

Following understanding their preferences we were interested in exploring participants' expectations from researchers for the return of individual genetic results. 


\section{Why_participants expect results}

Participants described three overarching reasons for why they expected researchers to share results, namely because this is a sign of respect, because they have a moral obligation to do so, and because feedback could promote long-term trust between participants and researchers.

\section{Respect and a moral obligation to return results}

Many participants emphasised that they consider a return of individual genetic results a demonstration of respect from researchers towards participants. Some even elaborated that it should be considered a moral or ethical obligation for researchers to return individual genetic results which may empower participants. See,

"Yes, I do, because I think ethically, I think it's the right thing to do. Because if I think, if I have the information, and I can better someone's life, or something that I can apply that works for me and someone else can learn, why not. You see? So regardless of the long process of going down all those numbers and going back to the root of it, I think you should still make that effort". (DFDG 2: Mixed Ancestry parent)

A few participants suggested that researchers ought to also assist individuals in getting more information about the condition which they may be predisposed to getting and/or provide recommendations about specific resources which may be helpful for them. For example, Interviewer: What you are saying is that they should call that person in and give them advice?

Parent: I am saying that the researcher should not stop only with the study, but also to continue looking for ways to help the individual. (DFGD 4: Xhosa parent)

And another,

"... The only thing that was on my mind about the session from last time, was the moral obligation that the researchers or doctors, whoever is sitting with the information has. They should have a moral obligation to let the patients know." (DFDG 2: Mixed Ancestry parent)

\section{Building Trust}

Many participants also alluded that receiving their individual genetic results may build trust between research participants and researchers.

"The NeuroDEV researchers did explain to us, there is a part, like that lady said, it's not like a must for them to let us know... but I think they should use their own initiative at that time, if they find something else and just, you know, not act like a doctor or a researcher, but act like a person and just tell that person." (DFDG 1: Mixed Ancestry parent) 
One participant suggested that in the event that researchers do not share individual results and the individual learns that that information may have allowed them to prevent or reduce the risk of getting the disease, they may end up blaming the researchers which could undermine the trust between researchers and participants. For example,

"As researchers you have to keep such a person in the loop because you will never know at the end if they will blame you or not. They might say that you knew about their condition and did nothing. The blame will be on you as researchers. Let the person be the one that has the responsibility to take action." (DFGD 4: Xhosa parent)

Another person specified that it would be important for researchers to feedback genetic information that is helpful for the individual. Even if the genetic information is about an untreatable, unpreventable disease, these participants emphasised that researchers should have an obligation to return that information and not doing so could threaten the trust between the two parties.

"Only if they have information that can help you. I think that they should have an obligation then, and also ethically they should do what is right, if they have that information. But if it cannot help you, and there's nothing that they can do about it, they should still give you that information and let the person decide for themselves. That is what I think."(DFDG 2: Mixed Ancestry parent)

\section{Discussion}

Our findings suggest that most participants would prefer to receive individual genetic results across all four categories investigated in this study, regardless of severity, actionability and preventability. There were a few exceptions however, with a few participants indicating uncertainty about wanting to receive results or a desire not to know due to concerns about possible psychological harm such as increased anxiety. This was particularly true for receiving results which are not medically actionable. Those who wanted to receive results emphasised that it would be preferable that results for severe conditions be delivered in conjunction with referral information and/or in the presence of a counsellor. Reasons for wanting genetic risk information included, 1) genetic information could empower or emancipate individuals and 2) it could improve lifestyle and behavioural decisions. We found that the reasons for expecting genetic results were, 1 ) receiving results would be a demonstration of respect from researchers and it is their moral obligation, and 2) it would build trust between genomics research participants and researchers. Generally, we found that participants were most interested in results which might have clinical actionability (through prevention or treatment), personal utility (psychological and behaviour preparedness) or which could inform future reproductive decisions (having more children) (1).

Our results are consistent with those from international research which found potential or enrolled genomic research participants to have a high interest in receiving individual genetic results for medical, familial, reproductive and/or personal reasons $(8,11,14,24-27)$. For example, in Sanderson and colleagues's study, most individuals from the general public wanted to receive individual genetic results for reasons such as, 'to motivate changes in lifestyle, to seek medical intervention, to prepare for the 
future, for curiosity, interest in genetics, to provide risk information to other family members, as well as for their future potential children and family planning'(11). Similarly, participants in their study also expressed concern for possible psychological impacts due to receiving genetic results. Our findings are also consistent with views of parents of children enrolled in cancer genomics research, which found parents to be overwhelmingly interested in results of theirs or of their children's that represented a predisposition to disease (13). With regards to expectations of participants, previous research has also found that the majority of participants expect to receive results from genetic studies (12) and researchers have suggested that considering participants' expectations is important for cultivating lasting trustbuilding between research participants and researchers (28).

Possible reasons for the overwhelming interest in receiving individual genetic results among our participants may be, 1) their primary interest in understanding the influence of genetics in their children's development of their neurodevelopmental condition, 2) they may be conflating diagnostic genetics testing with genetics research , 3) some may have strictly associated genetic results with common diseases such as cancer and 4) many participants may just want any health-related information which they are unlikely to get otherwise, given the limited capacity and resources in South Africa's public healthcare system. The last reason is particularly important because it relates to issues of social inequality in South Africa. The realities of living in low-resourced poverty-stricken environments, where it is difficult to assess healthcare, may be informing a desire to receive genetic information. The hope of our participants may be that receiving individual genetic information may in some way contribute to changing their health trajectories. There is considerable push-back from researchers on that point (1), with the most notable objections being the uncertainty of significance for results from genomics research which are not diagnostic and which may not be as useful to participants as they hope them to be.

Overall, findings from our study suggest the question about whether to return individual genetic results from a research context needs to be addressed openly and swiftly with participants at all stages of the research process, from the consent process onwards. We found that despite knowing that the genomics research project they participated in will only be returning pertinent findings, participants generally have an increased interest and high expectations of receiving all incidental results from genomics studies. Addressing this question early in the research process may manage participant's expectations. It is equally important that during the consenting stage that researchers explain the limitations of clinical and personal utility of individual genetic results. And in the event of an incidental finding not discussed during the initial consent, our participants emphasised the importance of re-contacting the participant and giving them an option to consent to receiving feedback on such a result.

Based on the low-levels of formal education and limited exposure to genetic literacy among many African participants (29-31), our results suggest that it would be important for African researchers to first educate participants on genomics research, particularly in cases where they have to consent to either be recontacted or not for incidental findings (32) and encourage realistic expectations of the clinical and personal utility of any individual results prior to asking for their views on this ethically important question. 
Taken together, our study suggests that in the context of African genomics research it may be important to consider participants' views on feedback of genetic results which would likely empower or emancipate individuals, families and communities. In doing so, genetic literacy is an important factor to consider. Our study revealed that educating participants about genetics and genomics research is an important step to ethically engaging with participants on important topics such as return of results, which could potentially impact the direction of future genomics research. Additionally, the deliberative focus group methodology is a useful way of fostering nuanced deliberations between research participants and the researchers which challenge participants to think deeply about these issues while enhancing the depth of these discussions.

\section{Conclusion}

Our study provides some initial empirical evidence on the views of African participants regarding the return of individual genetic research results. These views may be important for African genomics researchers who are grappling with the inevitability of uncovering individually relevant genomic findings and considering whether these results should be fed back to participants. It would be particularly important to consider these findings in the development of best practices for the return of individual genetic results not only for the ethical return of results but also for the development of contextually relevant Africa-specific feedback policies.

\section{Strengths and Limitations}

This study has some limitations and strengths. First, this study utilized a small sample from a specific population (parents of children with neurodevelopmental disorders) and it is possible that their views on wanting to receive feedback may be impacted by their desire for any genetic information due to uncertainties about their children's conditions. Second, the qualitative nature of the research means that results can not be generalised across contexts. Third, the use of hypothetical case studies could be a limitation in that it may yield unrealistic responses as participants could be uncertain of what they would do in a hypothetical situation and not be able to relate. However, it could also be a strength in that the use of hypothetical scenarios means participants do not have to speak from their own situation, thus making it easier for them to share. Fourth, the deliberative method chosen in the conducting of the study, is another contributing strength because it allows for the educating of participants about complex concepts such as genetics and genomics before asking them their opinions and provided a learning platform that many parents of children with neurodevelopmental conditions were seeking. Obviously, there is a risk that the learning platform could be a source of bias. To reduce this risk, when developing the materials we used for the dFGDs (brochures, a video and a manual containing all explanations) we drew on our links with genetic counsellors and medical genetic professionals, both of whom are used to providing information about genetics and health risks in a non-directional manner.

\section{Declarations}


Ethics approval and consent to participate Ethics approval was obtained from UCT's Faculty of Health Sciences Human Research Ethics Committee prior to commencement of the study (FHS: 782/2018). All participants in this study provided written informed consent.

Consent for publication: Not applicable

Availability of data: The datasets used and/or analysed during the current study are available from the corresponding author on reasonable request.

Competing interests: The authors declare no conflict of interests.

Funding sources: This work was part of the IFGENERA study which is supported by the NIH Common Fund H3Africa Initiative: Award number U54HG009790 to AW and JdV. The work was also part of the NeuroDev project which is supported by the Stanley Center for Psychiatric Research at the Broad Institute and the NIMH: Award number U01MH119689 to KAD.

Author contributions: JdV and AW conceived the notion of this project and sourced funding for the research. OPM and MCF developed the tools for the study and led the data collection process. All authors discussed the findings and data analysis strategy. OPM led data analysis with support from CAA and MCF; OPM and CAA jointly coded the data and developed the hierarchical coding scheme with support from JdV. The entire study team discussed emerging insights. OPM and CAA developed the first draft of the manuscript; all authors read and contributed to the draft manuscript.

Acknowledgements: We would like to gratefully acknowledge and thank the NeuroDEV project coordinator, Emma Eastman who assisted with identifying participants and sites for recruitment for this study, Lebogang Montewa in the IFGENERA team who assisted us with data collection logistics as well as all our participants who generously shared their views with us.

Authors information: OPM is a postdoctoral research fellow in the Department of Medicine at the University of Cape Town. CAA is a research assistant in the Department of Medicine at UCT. CAA is also an MPH graduate. MCF and DR are PhD candidates in the Department of Medicine at UCT. KAD is an Associate Professor in Paediatrics. KAD is also the Head of the Division of Developmental Paediatrics, in the Department of Paediatrics and Child Health and the Deputy Director of the Neuroscience Institute at UCT. AW is a medical geneticist and a Professor of Genetics in the Division of Human Genetics, in the Department of Pathology \& Institute of Infectious Disease and Molecular Medicine at UCT. AW is also the Deputy Director of Research in the Faculty of Health Sciences at UCT. JdV is an Associate Professor in the Department of Medicine at UCT. JdV is also a Chief researcher in Bioethics and the founding Chair of the H3Africa Ethics Working Group.

\section{References}


1. Thorogood A, Dalpé G, Knoppers BM. Return of individual genomic research results: are laws and policies keeping step? Eur J Hum Genet. 2019;27(4):535-46.

2. Kalia SS, Adelman K, Bale SJ, Chung WK, Eng C, Evans JP, et al. Recommendations for reporting of secondary findings in clinical exome and genome sequencing, 2016 update (ACMG SF v2. 0): a policy statement of the American College of Medical Genetics and Genomics. Genet Med. 2017;19(2):249-55.

3. Popejoy AB, Fullerton SM. Genomics is failing on diversity. Nature. 2016;538(7624):161.

4. Wonkam A, de Vries J. Returning incidental findings in African genomics research. Nat Genet. 2020;52(1):17-20.

5. Rutakumwa R, de Vries J, Parker M, Tindana P, Mweemba O, Seeley J. What constitutes good ethical practice in genomic research in Africa? Perspectives of participants in a genomic research study in Uganda. Global Bioethics. 2019:1-15.

6. H3Africa. H3Africa Guideline for the Return of Individual Genetic Research Findings. 2018.

7. Klitzman R, Appelbaum PS, Fyer A, Martinez J, Buquez B, Wynn J, et al. Researchers' views on return of incidental genomic research results: qualitative and quantitative findings. Genet Med. 2013;15(11):888-95.

8. O’Daniel J, Haga S. Public perspectives on returning genetics and genomics research results. Public health genomics. 2011;14(6):346-55.

9. Middleton A, Morley KI, Bragin E, Firth HV, Hurles ME, Wright CF, et al. Attitudes of nearly 7000 health professionals, genomic researchers and publics toward the return of incidental results from sequencing research. Eur J Hum Genet. 2016;24(1):21-9.

10. Shalowitz DI, Miller FG. Communicating the results of clinical research to participants: attitudes, practices, and future directions. PLoS Med. 2008;5(5):e91.

11. Sanderson SC, Linderman MD, Suckiel SA, Diaz GA, Zinberg RE, Ferryman K, et al. Motivations, concerns and preferences of personal genome sequencing research participants: baseline findings from the HealthSeq project. Eur J Hum Genet. 2016;24(1):14-20.

12. Facio FM, Eidem H, Fisher T, Brooks S, Linn A, Kaphingst KA, et al. Intentions to receive individual results from whole-genome sequencing among participants in the ClinSeq study. Eur J Hum Genet. 2013;21(3):261-5.

13. Fernandez CV, Bouffet E, Malkin D, Jabado N, O'Connell C, Avard D, et al. Attitudes of parents toward the return of targeted and incidental genomic research findings in children. Genet Med. 2014;16(8):633-40.

14. Sabatello M, Zhang Y, Chen Y, Appelbaum PS. In Different Voices: The Views of People with Disabilities about Return of Results from Precision Medicine Research. Public Health Genomics. 2020;23(1-2):42-53.

15. Lakes KD, Vaughan E, Lemke A, Jones $M$, Wigal T, Baker $D$, et al. Maternal perspectives on the return of genetic results: context matters. Am J Med Genet A. 2013;161(1):38-47. 
16. Marsh V, Kombe F, Fitzpatrick R, Williams TN, Parker M, Molyneux SJBme. Consulting communities on feedback of genetic findings in international health research: sharing sickle cell disease and carrier information in coastal Kenya. BMC Med Ethics. 2013;14(1):1-13.

17. Ewuoso C. Ubuntu philosophy and the consensus regarding incidental findings in genomic research: a heuristic approach. Med Health Care Philos. 2020.

18. De Menil V, Hoogenhout M, Kipkemoi P, Kamuya D, Eastman E, Galvin A, et al. The NeuroDev Study: Phenotypic and Genetic Characterization of Neurodevelopmental Disorders in Kenya and South Africa. Neuron. 2019;101(1):15-9.

19. Holm IA, Iles BR, Ziniel SI, Bacon PL, Savage SK, Christensen KD, et al. Participant satisfaction with a preference-setting tool for the return of individual research results in pediatric genomic research. Journal of Empirical Research on Human Research Ethics. 2015;10(4):414-26.

20. Gale NK, Heath G, Cameron E, Rashid S, Redwood S. Using the framework method for the analysis of qualitative data in multi-disciplinary health research. BMC Med Res Methodol. 2013;13(1):1-8.

21. Braun V, Clarke V. Thematic analysis. In: Cooper H, editor. APA Handbook of Research Methods in Psychology. 2: American Psychological Association; 2012.

22. Siccama CJ, Penna S. Enhancing validity of a qualitative dissertation research study by using NVivo. Qualitative Research Journal. 2008;8(2):91-103.

23. Meel B. Witchcraft in Transkei region of south African: case report. Afr Health Sci. 2009;9(1):61-4.

24. Allen NL, Karlson EW, Malspeis S, Lu B, Seidman CE, Lehmann LS, editors. Biobank participants' preferences for disclosure of genetic research results: perspectives from the OurGenes, OurHealth, OurCommunity project. Mayo Clin Proc; 2014: Elsevier.

25. Kaphingst KA, Ivanovich J, Lyons S, Biesecker B, Dresser R, Elrick A, et al. Preferences for learning different types of genome sequencing results among young breast cancer patients: Role of psychological and clinical factors. Transl Behav Med. 2018;8(1):71-9.

26. Wynn J, Martinez J, Duong J, Chiuzan C, Phelan JC, Fyer A, et al. Research participants' preferences for hypothetical secondary results from genomic research. J Gen Couns. 2017;26(4):841-51.

27. Murphy J, Scott J, Kaufman D, Geller G, LeRoy L, Hudson K. Public expectations for return of results from large-cohort genetic research. The American Journal of Bioethics. 2008;8(11):36-43.

28. Kraft SA, Cho MK, Gillespie K, Halley M, Varsava N, Ormond KE, et al. Beyond consent: building trusting relationships with diverse populations in precision medicine research. The American Journal of Bioethics. 2018;18(4):3-20.

29. Faure MC, Matshabane OP, Marshall P, Appelbaum PS, Stein DJ, Engel ME, et al. Does genetics matter for disease-related stigma? The impact of genetic attribution on stigma associated with rheumatic heart disease in the Western Cape, South Africa. Soc Sci Med. 2019:112619.

30. Matshabane OP, Campbell MM, Faure MC, Marshall PA, Mayosi BM, Stein DJ, et al. Exploring how a genetic attribution to disease relates to stigma experiences of Xhosa patients with schizophrenia in South Africa. Soc Psychiatry Psychiatr Epidemiol. 2020. 
31. Tindana P, Bull S, Amenga-Etego L, de Vries J, Aborigo R, Koram K, et al. Seeking consent to genetic and genomic research in a rural Ghanaian setting: a qualitative study of the MalariaGEN experience. BMC Med Ethics. 2012;13(1):1-12.

32. Appelbaum PS, Waldman CR, Fyer A, Klitzman R, Parens E, Martinez J, et al. Informed consent for return of incidental findings in genomic research. Genet Med. 2014;16(5):367-73. 